East African Medical Journal Vol. 81 No. 4 April 2004

ENDOSCOPIC VARICEAL BAND LIGATION: A LOCAL EXPERIENCE

P. G. Jani, MMed., FRCS., Associate Professor, Department of Surgery, College of Health Sciences, University of Nairobi, P.O. Box 45640-00100, Nairobi, Kenya

\title{
ENDOSCOPIC VARICEAL BAND LIGATION: A LOCAL EXPERIENCE
}

P. G. JANI

\begin{abstract}
Objective: To evaluate the results of endoscopic variceal band ligation (EVBL) in the local set-up.

Design: Retrospective analysis of data of all patients who had EVBL.

Setting: Patients having EVBL at the office endoscopy suite. The Nairobi Hospital, the Aga Khan Hospital and M.P Shah Hospital.

Methods: The varices were diagnosed at oesophagogastroduodenoscopy (OGD) in patients with hemetemesis and EVBL performed after xylocaine throat spray and intravenous pethidine and bendiazepam sedation. Majority of the patients had EVBL performed on an outpatient basis on weekly intervals until variceal obliteration.

Results: A total of 43 patients underwent 119 EVBL sessions at which 523 varies were banded. Four patients had active bleeding and the rest had EVBL for secondary prophylaxis. The variceal kill time was shorter and the transfusion requirements were less and none of the patients developed oesophageal strictures.

Conclusion: Endoscopic variceal band ligation was noted to give better results in the management of patients with oesophageal varices in the local set-up, when compared to sclerotherapy in the past.
\end{abstract}

\section{INTRODUCTION}

Variceal bleeding is an important cause of upper gastrointestinal bleeding. It is characteristically abrupt and massive with an accompanying high mortality. The first episode of variceal bleeding is not only associated with a high mortality but also with a high recurrence rate in those who survive(1). Therefore different therapeutic strategies have focussed on preventing the first episode of variceal bleeding (primary prophylaxis), to control haemorrhage during the acute variceal bleeding (emergency treatment), and to prevent re-bleeding (secondary prophylaxis). The modalities used for the management of oesophageal varices include pharmacological(2-4), endoscopic(5-7), surgical(8-10), interventional radiology $(8,11)$ and laser therapy(12).

Endoscopic therapy, in places where the expertise is available has played a major role in the management of patients with variceal pathology for the past three decades. Sclerotherapy was the first endoscopic modality followed by EVBL, which has been available for a decade now. During the early years of its introduction EVBL was mainly advocated for secondary prophylaxis but in the recent past EVBL has also established a firm position in the management of emergency bleeding with superior results to other forms of therapy(13). For primary prophylaxis of variceal bleeding medical therapy is usually advocated but recent studies indicate that EVBL also reduces the frequency of the first variceal bleed and bleeding related mortality(14). EVBL may therefore prove to be the optimal endoscopic method of treating oesophageal varices. Over the years EVBL has also resulted in a shorter variceal kill time when compared to sclerotherapy and this translates into decreased costs, a point very crucial in developing countries(15). EVBL is also shown to be superior in terms of re-bleeding occurring less commonly and transfusion requirements being lower. Finally complications with EVBL are much fewer when compared to sclerotherapy(5).

EVBL with the use of an overtube has been reported to result in proximal oesophageal mucosal shearing with bleeding but this is very rare and can be avoided with gentle insertion of the overtube(16). The use of the overtube can also be dispensed with completely when a multifire ring applicator is used(17).

EVBL has also been successfully used in the paediatric age group with good results $(8,9)$. EVBL was first practiced in Kenya in 1995 prior to which sclerotherapy was the mainstay of endoscopic management of oesophageal varices. During the initial period, EVBL was mainly performed for secondary prophylaxis after the index bleed and sclerotherapy was the mainstay of treatment for acute bleeds. However with increasing experience, EVBL is now also used for emergency treatment of acute bleeds. EVBL is not used for primary prophlylaxis in the local set up as yet.

\section{MATERIALS AND METHODS}

Endoscopic variceal band ligation was first introduced for the management of oesophageal varices in 1995. Forty three patients have had EVBL using an endoscopic rubber band ligator (Bard interventional products, mass). The varices were diagnosed at OGD in patients who had presented with hemetemesis, and found to have oesophageal varices as the cause of their bleed. Majority of the patients had EVBL performed for secondary 
proplylaxis and on an out patient basis. Four patients presented with active bleeding and were treated as inpatients. Two of them, in the initial period were controlled with sclerotherapy and the other two had successful EVBL therapy.

EVBL for active bleeding was only tried after gaining adequate confidence and experience. In all the patients, the endoscopy was performed under diazepam and pethidine sedation given intravenously after xylocaine throat spray. The overtube was positioned over the scope, and a complete examination of the oesophagus (noting the extent and grade of varices), stomach and duodenum performed. The overtube was then positioned across the cricopharyngeal sphincter and the scope withdrawn. The endoscopic rubber band ligator adaptor was then loaded onto the scope, passed through the overtube and a varix over the lower $5 \mathrm{cms}$ of the oesophagus sucked and banded. The scope was withdrawn, loaded and between one to six bandings performed at each session. The patient was then observed until awake and allowed home with a relative.

The interval between sessions varied from five days to two weeks according to the probability of re-bleeding. After complete obliteration of varices, the patients were followed up at three monthly intervals initially, and later yearly.

\section{RESULTS}

Endoscopic variceal band ligation (EVBL) was carried out in 43 patients of whom 32 were males and 11 females. The age ranged between 15 and 73 years. A total of 119 EVBL sessions were carried out and between 2 to 6 rings were fired at each session depending on the patients condition, size of varices, probability of bleeding and re-bleeding and complications related to the procedure. A total of 523 varices were banded. One patient developed stridor upon placement of the overtube and hence could not be banded, and all the other patients tolerated the overtube without any complications.

Of the 43 patients, four were treated for active bleeding. Two of these had sclerotherapy performed (in the early part of the series) for emergency treatment followed by EVBL for secondary prophylaxis. Two other patients with active bleeding had successful EVBL after enough experience had been attained. The other 39 patients had EVBL for secondary prophylaxis and most of these procedures were performed on an outpatient basis.

There were two patients who bled during EVBL for secondary prophylaxis and both had successful arrest with sclerotherapy combined with EVBL.

The varices extended over the entire oesophagus in seven patients, over the mid and lower one third in 28 patients and over the lower third only in eight patients, although a considerable overlap existed in many patients. Of the 43 patients who had EVBL for secondary prophylaxis eventually, 33 had Grade IV varices and 10 had Grade III varices. Of the four patients who had emergency treatment, three had Grade IV varices while one had Grade III varices.

Not all the patients could afford all the investigations to determine the exact cause of their portal hypertension. Only 20 of the 43 patients had investigations which showed 11 patients with schistosomal fibrosis, eight with cirrhosis (four most probably alcoholic and four post hepatitic) and one had idiopathic portal hypertension. The variceal kill time with EVBL was recognised to be definitely shorter as compared to sclerotherapy practiced in the past, but no statistical analysis was performed. On an average a patient with Grade IV varices had a variceal kill time of four EVBL sessions.

Only two of the 43 patients, who had EVBL for secondary prophylaxis and bled, required to be transfused and this showed that the transfusion requirements are much lower with EVBL.

Re-bleeding was also lower and occurred in only two of the 43 patients. None of the patients who had EVBL performed developed oesophageal ulcers or strictures.

\section{DISCUSSION}

Variceal bleeding is a major cause of upper gastrointestinal bleed in the local set-up. Portal hypertension secondary to schistosomal fibrosis is common since schistosomasis is endemic in both Nyanza and Eastern provinces. The management of oesophageal varices at present is focussed mainly with the bleeding aspects of varices. Maybe, in future, with further medical progress the focus will be towards the control of intravariceal pressure and variceal size which in turn will diminish the problems of variceal bleeding altogether. Variceal bleeding is managed from three different aspects (i). Primary prophylaxis, (which is the primary prevention of variceal bleeding) (ii) Emergency treatment of actively bleeding varices. (iii) Secondary prophylaxis (which is prevention of recurrent variceal bleeding).

Primary prophylaxis is mainly performed with medical treatment at present using Beta blockers and nitrates with the aim of vasodilating the splanchnic circulation and possibly decreasing the effects of collaterals that develop due to portal hypertension(18). Trials on primary prophylaxis with EVBL have shown promising results(1923) and maybe EVBL will establish a firm role in future, for primary prophylaxis, especially in centres where experience with, EVBL has been attained. Locally EVBL for primary prophylaxis is not practised, as yet.

The recommendations for the management of emergency treatment of variceal bleeding during the early period of EVBL were strongly in favour of sclerotherapy. However most centres after having attained adequate experience with EVBL for secondary prophylaxis, now report better results with EVBL for emergency bleeding than sclerotherapy $(19,24)$. In the present series two patients had successful arrest of active bleeding with EVBL. Since majority of the patients with varices present after the bleeding has stopped, gaining experience with EVBL for emergency bleeds takes long.

The greatest experience with EVBL is for secondary prophylaxis. EVBL is now accepted as the gold standard endoscopic therapy for recurrent variceal haemorrhage(25). In the present series all the 43 patients had EVBL performed for secondary prophylaxis, and were managed by the sole author. Paediatric patients were not included. The number of varices banded at any one session depended on the number of variceal columns present and upto six rings were used for banding. In actively bleeding patients, varices at the lower oesophageal junction were banded or sclerosed, lower oesophageal if no clear bleeding varix could be identified. In patients with large varices and red signs of impending rupture only the life threatening varices 
were banded. In patients with large varices and no red signs, upto six bandings were performed and in patients with residual varices, after initial control, two to four rings were fired for the remaining varices. The aim of banding the varices, as above, was to arrest bleeding in the acute bleeders, prevent bleeding and stabilise patients at high risk of re-bleeding and obliterate all varices eventually.

With such a policy it was noted that emergency control of variceal bleeding was feasible, re-bleeding occurred less commonly, transfussion requirements were lower and the complications were minimal. Two of the patients in the early part of the series had bleeding during EVBL and this was thought to be due to banding of an incomplete loop of the varix. No patient in the later part of the series required any transfusions. The average variceal kill time for a patient with Grade IV varices was four sessions and with EVBL performed on an outpatient basis, resulted into better compliance with reduced costs to obliterate the varices. This fact is of the utmost importance in developing countries where resources are limited.

When comparing EVBL with sclerotherapy practiced in the past for six years, EVBL was felt to be a much better technique with regards to re-bleeding and transfusion requirements. Moreover the problem of oesophageal ulceration and strictures over the lower oesophagus experienced with sclerotherapy were not seen with EVBL. These results can be explained by the fact that with EVBL, a loop of the varix is banded by a rubber ring, completely occluding the varix, and not affecting the intervariceal mucosa of the oesophagus.

\section{ACKNOWLEDGEMENT}

To Dr. J. P. Jani for her assistance in preparation of this manuscript.

\section{REFERENCES}

1. Helmy, A. and Hayes, P.C. Current endoscopic therapeutic options in the management of variceal bleeding. Alimentary pharma. Therapeutics, 2001;15:575-594.

2. Banares, R., Albillos, A., R., Diego, A., et al. Endoscopic treatment versus endoscopic plus pharmacologic treatment for acute variceal bleeding: a meta-analysis. J. Hepatology. 2002; 35:609-615.

3. Moitinho, E. Planas, R. Banares, R. et al. Multicenter randomized controlled trail comparing different schedules of somatostatin in the treatment of acute variceal bleeding. J. Hepatology. 2001; 35:712-718.

4. Villanueva, C. Ortiz, J. Minana, J. et al. Somatostatin treatment and risk stratification by continuous portal pressure monitoring during acute variceal bleeding. Gastroenterology. 2001; 121:110-117.

5. Nevens, F. and Rutgeerts, P. Variceal band ligation in the management of bleeding oesophageal varices: an overview. Digestive and liver disease: official journal of the Italian Society of Gastroenterology and the Italian Association for the study of the liver. 2001; 33:284-287.

6. Hou, M. C. Chen, W.C. Lin, H.C. et al. A new "sandwich" method of combined endoscopic variceal ligation and sclerotherapy versus ligation alone in the treatment of esophageal variceal bleeding: a randomized trial. Gastrointestinal endoscopy. 2001; 53:572-578.

7. Bosch, J. The sixth Carlos E. Rubio Memorial Lecture.
Prevention and treatment of variceal hemorrhage. Puerto Rico hlth scin. J. 2000; 19:57-67.

8. Vladimir, K., Jiri, K., Roman, H., Rene, A. and Petr, U., Esophagogastric devascularization as the last option in the management of variceal bleeding. Hepatogastroenterology. 2002; 49:244-246.

9. Selzner, M. Tuttle-Newhall, J. E. Dahrn, F. Suhocki, P. and Clavien, P.A. Current indication of a modified Sugiura procedure in the management of variceal bleeding. J. Amer. College Surg. 2001; 193:166-173.

10. Hillebrand, D.J Kojouri, K. Cao, S. Runyon, B.A. Ojogho, and O. Concepcion, W. Smalldiameter portacaval H-graft shunt: a parpdigm shift back to surgical shunting in the management of variceal bleeding in patients with preserved liver function; Liver transplantation: official publication of the American Association for the Study of Liver Diseases and the International Liver Transplantation Society. 2000; 6:459-465.

11. Han, S.W. Joo, Y.E. Kim, H.S. et al. Clinical results of the transjugular intrahepatic portosystemic shunts(TIPS) for the treatment of variceal bleeding. Korean J. Inter. Med. 2000; 15:179-186.

12. Cipolletta, L. Bianco, M.A., Rotondano, et al. Argon plasma cougulation prevents variceal recurrence after band ligation of esophageal varices: Preliminary Results of a prospective randomised trial. Gastro. Endoscopy. 2002; 56:467-471 .

13. Vlavianos P., and Westaby D. Management of acute variceal haemorrhage. Euro. J. Gastro. Hepatology. 2001;13:335342.

14. Burroughs, A.K. and Patch, D. Primary prevention of bleeding from esophageal varices. New Eng. J. Med. 1999; 340; 1033-1035.

15. Jani, P.G. Oesophageal variceal banding: report of the first eight cases in Kenya. East Afr. Med. J. 1997; 74:395-396.

16. Hoepffner, N. Foerster, E. Menzel, J. Gillessen, A. and Domschke, W. Severe complications arising from oesophageal varix ligation with the stiegmann -Goff set. Endoscopy. 1995; 27:345.

17. Mckiernan, P.J. Beath, S.V. and Davison, S.M. A prospective study of endoscopic esophageal variceal ligation using a multiband ligator. J Pediatr Gastro. Nutr. 2002; 34:207-311.

18. Merkel, C. Bolognesi, M. Sacerdoti, D. et al. The hemodynamic response to medical treatment of portal hypertension as a predictor of clinical effectiveness in the primary prophylaxis of variceal bleeding in cirrhosis; Hepatology (Baltimore, Md.) 2000; 32:930-934.

19. Laine, L. and Cook, D. Endoscopic ligation compared with sclerotherapy for treatment of esophageal variceal bleeding. A meta-analysis. Ann. Intern. Med. 1995; 123:280-287.

20. Lo, G.H. Hwu, J.H. and Lai, K.H. Prophylactic banding ligation of high-risk esophageal varices-an interim report(abstract). Gastro. 1995; 108:A1112.

21. Sarin, S.K., Guptan, R.C. Jain, A.K. et al. A randomized controlled trial of endoscopic variceal band ligation for primary prophylaxis of variceal bleeding. Euro. J. Gastro. Hepatol. 1996; 8:337-342.

22. Chen, C.Y. and Chang, T.T. Prophylactic endoscopic variceal ligation (EVL) for esophageal varices (abstract). Gastroenterology. 1997; 112:A1240.

23. Lay, C.S. Tsai, Y.T. Teg, C.Y. et al. Endoscopic variceal ligation in prophylaxis of first variceal bleeding in cirrhotic patients with high-risk esophageal varices. Hepatology. 1997; 25:346-350.

24. Stiegmann, G.V. Goff, J.S. Michaletz-Onody, P.A. et al. Endoscopic sclerotherapy as compared with endoscopic ligation for bleeding esophageal varices. New Engl. J. Med. 1992; 326; 23:1527-1532.

25. Knechtle, S.J. and Rikkers, L.F. Current management of esophageal variceal bleeding; Advances in surg. 1999; 33:439-458. 